\title{
Book review: From the Yenisei to the Yukon: Interpreting Lithic Assemblage Variability in Late Pleistocene/Early Holocene Beringia
}

\author{
Jennifer Anderson \\ Texas State University - San Marcos, Department of Anthropology. 601 University Dr. San Marcos, Texas, \\ United States. Email: jba35@txstate.edu
}

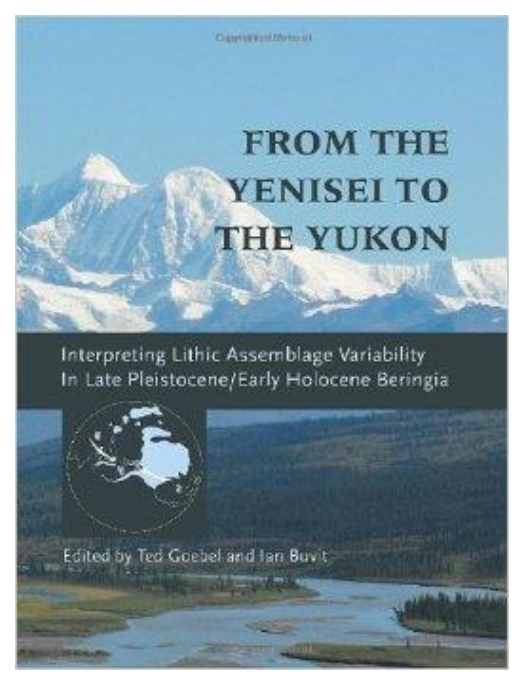

From the Yenisei to the Yukon: Interpreting Lithic Assemblage Variability in Late Pleistocene/Early Holocene Beringia

Edited by Ted Goebel and Ian Buvit

Texas A\&M University Press, 2011, pp. 394. ISBN 978-1-60344-321-0

http://www.tamupress.com/product/From-the-Yenisei-to-the-Yukon,6578.aspx

This volume represents the outcome of the "Explaining Lithic Assemblage Variability across Beringia" symposium, held at the 73rd Annual Meeting of the Society for American Archaeology. This symposium was a follow-up to a workshop held at the Centre for the Study of the First Americans, Texas A\&M University, in which researchers discussed the current state of knowledge surrounding the Pleistocene peopling of Beringia and acknowledged the need for future research methods to focus on interdisciplinary, multinational approaches. The twenty-one chapters in this volume directly reflect this endeavour, showcasing a diverse array of research that analyses lithic assemblage variability in late Pleistocene Beringia. Though

Published by the School of History, Classics and Archaeology, University of Edinburgh ISSN: 2055-0472. URL: http://journals.ed.ac.uk/lithicstudies/

This work is licensed under a Creative Commons Attribution 2.5 UK: Scotland License. 
individual chapters focus on questions pertaining to specific regions or assemblages in Beringia, collectively they address a conundrum that all lithic analysts face, that of understanding what variability within a given lithic assemblage represents.

In the introduction, the editors provide the reader with the historical framework for the development of the major, heretofore-disparate Beringian lithic technocomplexes. Chapter 2 Graf examines the coeval Afontova and Kokorevo technocomplexes of western Beringia, finding that the variability between the two was not sufficient to consider the two complexes distinct. In Chapter 4, two coeval microblade core reduction sequences were identified in the Lower Vitim Valley of Siberia. The first resembled the Upper Palaeolithic Yubetsu (Diuktai) tradition, given the presence of both wedge-shaped cores and prepared bifaces, while the second resembled the early Holocene Sumnagin tradition with prismatic cores. In Chapter 5, experimental pressure flaking data was compared to pressure flake debitage from Diuktai Cave to determine the type(s) of pressure flaking being used at the site.

Chapters 3 and 16 examine settlement mobility in Beringia. Buvit and Terry examined evidence for increased settlement mobility during the Last Glacial Maximum (LGM) by using the abundance of local raw materials in a given assemblage as a proxy for sedentism. They conclude that due to increased usage of non-local raw materials from the Middle Upper Palaeolithic to the Late Upper Palaeolithic (despite consistent access to locally abundant materials), mobility did seem to increase during the LGM. In Alaska, Reuther et al. determined that the earliest populations were exploiting local obsidian sources. This exploitation continued into the Holocene, when obsidian from these two sources was found in distant assemblages. Chapters 6, 7, and 10 examine the complex migration patterns of several lithic technocomplexes across northeast Asia and western Alaska. Wedge-shaped microblade cores, associated with the Diuktai culture, emerged from western Siberia. Although it has been assumed that these microblade traditions migrated into Alaska, no wedge-shaped microblade cores have yet been recovered from northeast Asia. In Chapter 10, however, Holmes cites the production and reduction of microblade cores by use of the Yubetsu technique at Swan Point as the best evidence for a trans-Beringian "land bridge" migration. This Diuktai-like microblade technology in Alaska became known as the Denali complex.

According to Ackerman (Chapter 15), Denali hunters used microblades as lateral insets for arrowheads in the earliest expression of a North American bow-and-arrow weapon system. In Chapters 8 and 13, analyses of land use show that microblade tools are most often associated with cold weather hunting. One possible reason for this could be that microblades emerged during harsh conditions of the LGM and re-emerged during subsequent cold climatic oscillations, indicating that microblade composite technology was better suited to handle cold weather conditions than brittle bifacial projectiles (Chapter 14).

The Nenana complex (Chapter 12) was a non-microblade lithic tradition that coexisted with the Denali complex in Alaska. Nenana toolkits contained teardrop-shaped Chindadn projectile points, resembling interior North American lithic traditions more so than Asian ones. Similarly, Hoffecker associated the Mesa complex of eastern Beringia (Chapter 9), which contained lanceolate projectile points and was associated with bison hunting, with lithic traditions of the North American Plains. Chapters 17, 18, and 19 examine the expansion of the Denali and Chindadn/Nenana complexes into western Canada.

The remaining chapters offer thought-provoking syntheses by Dumond and Dixon. Dumond's slightly contrarian (his words) synthesis is a breath of fresh air for those of us who remain unconvinced of the first American's Asian progenitor. He points out the tenuous connections between Nenana and Clovis cultures and their Asian antecedents. Dixon's synthesis acknowledges the many difficulties in first identifying, then analysing and interpreting, lithic assemblage variability. His parting words remind us all that although these analyses seem arduous, progress is never hopeless. Certainly this volume attests to that. 